\title{
New Parameters and Metrics for Multiparty Communications
}

\author{
Lei Liang, Zhili Sun, and Dan He* \\ CCSR, University of Surrey, UK \\ Email: L.liang@surrey.ac.uk
}

\begin{abstract}
All IPPM QoS parameter metrics are defined for endto-end one-way measurement. These metrics provide exact indicators of network layer quality in the pair communications. However, further attention should be put on the multiparty communications, which might use multicast routing protocols, e.g. the IP conferencing services, online gaming, online stock market and etc. To qualify the multiparty communications, the future QoS study and metrics are required to be defined. The purpose of this paper is to highlight the new QoS requirements of the multiparty communication services in terms of relative delay, relative jitter and relative packet loss. A set of parameter metrics is derived from the existing one-way metrics in IP Performance Metrics (IPPM) for the multiparty communications to present these new requirements. These parameter metrics are supposed to provide methods and rules for engineers to measure and judge the QoS of the multiparty communications.
\end{abstract}

Keywords - multiparty communication, measurement, QoS, parameter, metric

\section{INTRODUCTION}

Traffic measurement is crucial to the traffic engineering function. It is the ground to have the insight of the network operation state and problem anticipation. It is also crucial for optimizing the network because it can provide the feedback data for the engineer to adaptively optimize network performance in response to events and stimuli originating within and outside the network. It is essential to determine the quality of network services and to evaluate the effectiveness of traffic engineering policies. And experience indicates that measurement is most effective when acquired and applied systematically.

Traffic measurement has been playing a crucial role for the one-to-one communications in the history. Now it will continue this key role in the design and evaluation of the new network services, multiparty communications. These services are designed to enable more than two people to communicate with each others using VoIP technologies, e.g., IP conferencing, online gaming, online stock market and so on. Multiparty communications attend to use multicast routing protocols to make it feasible at network level. However, there is another key aspect we should mention here, the QoS. The VoIP application will not win its final success in the fighting with the traditional telephony services without an acceptable
QoS. It will be necessary to carry measurements in those multiparty communication systems to provide solid inputs to evaluate the QoS performance as well as appropriately present those results in terms of some well defined parameters.

To adopt traffic measurement technologies from the one-toone communications to multiparty communications, we will need to identify any different QoS requirements between them. The multiparty communications have additional QoS requirements to one-to-one communications. The latter only have requirements on the absolute QoS while the former one needs additional relative QoS support. The relative QoS requirements come from the nature of the group communications. It means the difference of absolute QoS between all users in a group. If some of users suffer worst absolute QoS than the rest, and if the difference between these two QoS levels is beyond a certain threshold, the multiparty communication might not be able to continue. Therefore, we need something to describe this QoS difference and present the requirements on the relative QoS.

This paper will introduce the existing standardized IPPM parameters and metrics designed for one-to-one communications first. And then we will propose a new set of parameters and their metrics derived from those one-to-one parameters. The calculation of the proposed parameters and possible measurement errors will be discussed.

\section{ONE-TO-ONE QOS PARAMETERS}

To determine what parameters are needed to measure is the most important factor before one launch his measurement. It is the key for choosing measurement tools, methodologies and accuracy. The Internet Engineering Task Force (IETF) IP Performance Metrics (IPPM) working group developed a set of standard metrics that can be applied to the quality, performance, and reliability of Internet data delivery services. Another working group named Benchmarking Methodology (BMWG) made a series of recommendations concerning the measurement of the performance characteristics of various internetworking technologies, which includes terminology, identifying a set of metrics that aid in the description of traffic characteristics, and methodology, required to collect said metrics. Additionally, the ITU-T Working Group T1A1.3 made similar network performance parameter definitions [1]. 
The IPPM developed a set of measurement parameters as well as the correspondent measurement methodologies with the cooperation with other relative working group such as BMWG, TEWG, ITU-T SG 12 and SG 13. Those parameters include:

1. Connectivity: If a packet transmitted from source A to destination $\mathrm{B}$ at time $\mathrm{T}$ can arrive $\mathrm{B}$, it is said that A has the connectivity to $\mathrm{B}$ at time $\mathrm{T}$.

2. One-way delay: The difference between the time when the source sends out the first bit of the packet and the time when the destination receives the last bit of the packet

3. One-way loss: If a packet transmitted from source A to destination $\mathrm{B}$ cannot arrive $\mathrm{B}$ in a certain time threshold, it is said that this packet is lost

4. Round-trip delay: The sum of the times needed for a test packet travel from source A to destination B and from B back to A.

5. One-way delay variation: The difference of the oneway delays of a selected pair of packets in the stream going from source A to destination B.

6. Loss patterns: The packet loss distribution.

7. Bulk transport capacity: The expected long term average data rate (bits per second) of a single ideal TCP implementation over the path in question.

The IPPM defined a general framework [2] for particular parameter metrics that can be deployed to gain common understanding by Internet users and Internet providers of the performance and reliability both of end-to-end paths through the Internet and of specific 'IP clouds' that comprise portions of those paths. The term "metric" is defined as a carefully specified quantity that is relative to the Internet performance and reliability people is interested in. It recommends defining particular metrics under some criteria and disciplines in order to allow people to speak clearly about Internet traffic performance. In several IETF meetings criteria for these metrics have been specified as follow:

1. These metrics must be concrete and well defined,

2. A methodology for a metric should have the property that it is repeatable: if the methodology is used multiple times under identical conditions, it should result in consistent measurements,

3. The metrics must exhibit no bias for IP clouds implemented with identical technology,

4. The metrics must exhibit understood and fair bias for IP clouds implemented with non-identical technology,

5. The metrics must be useful to users and providers in understanding the performance they experience or provide,

6. The metrics must avoid inducing artificial performance goals.
IPPM gives 6 sets of standardized metrics for the following parameters under the above criteria:

1. Metrics for measuring connectivity [3]

2. One-way delay metric [4]

3. One-way packet loss metric [5]

4. Round-trip delay [6]

5. One-way loss pattern [7]

6. Packet delay variation [8]

Each of these metrics is normally defined with three sections including metric name, metric parameters and metric units. The metric name contains basic information of the measurement such as packet type, unidirectional or bidirectional, and parameter name. The metric parameter section defines what traffic parameters should be recorded in the metric that can be used for further analysis. The metric unit part describes the unit type of the metric. For instance, the one-way delay metric is named "Type-P-One-way-Delay" that means packets measured in this metric are all type $P$ packet where $\mathrm{P}$ could be any protocols such as TCP, UDP and ICMP. Its metric parameters are $S r c$, the IP address of the packet source, Dst, the IP address of the packet destination, and $T$, the time the source sent out the first bit of the type $\mathrm{P}$ packet.

Correspondent to each metric, at least one measurement methodology is defined to acquire data from the network. These methodologies should have the property that it is repeatable: if the methodology is used multiple times under identical conditions, it should result in consistent measurements or continuity results with small variations.

These traffic parameters and their measurement methodologies were defined by IETF for the purpose of network performance and reliability analysis. They are vital for the network evaluation, especially QoS evaluation. I will introduce some of these metrics that are relevant to my later work one by one in the following paragraphs.

One-way delay: The definition of one-way delay of a packet is the difference between the time when the source sends out the first bit of the packet and the time when the destination receives the last bit of the packet (whenever a time, i.e., a moment in history, is mentioned in this document, it is understood to be measured in seconds (and fractions)) [4].

Packet delay variation (jitter): The one-way delay variation of a pair of packets within a stream of packets is defined as the difference of the one-way delays of a selected pair of packets in the stream going from measurement point (MP) 1 to measurement point MP2[8].

Round-trip delay: The round-trip delay is defined as the sum of the times needed for a test packet travel from the source to the destination and from the destination back to the source [6]. One-way packet loss: If a test packet does not arrive at its destination in a threshold, it is defined lost [5]. 


\section{PROPOSED QOS PARAMETERS FOR MULTIPARTY MULTIMEDIA COMMUNICATIONS}

All IPPM QoS parameters are defined for one-to-one connections. Further attention should be put on the multiparty applications that use multicast routing protocols, e.g. the IP conferencing services. The basic consideration is that in the multiparty communication, we have a group of people involved in the action rather than two. One may say that no matter how many people join the communication, the connections can still be treated as a set of end-to-end connections. However, we might not describe a multiparty communication by a set of end-to-end measurement metrics both because of the difficulty for understanding and the lack of convenience. For instance, an engineer might not describe the connections of a multiparty online conference in terms of one-way delay for user $\mathrm{A}$ and $\mathrm{B}, \mathrm{B}$ and $\mathrm{C}$, and $\mathrm{C}$ and $\mathrm{A}$ because people might be confused. And he might use the endto-end metrics with the worst and the best value to give users an idea of the QoS range of the service they are providing. But it's not clear enough and might not be accurate in a big multiparty communication. My suggestion is to use a more sophisticated way after reasonable mathematic deriving, i.e. mean, variation etc. the new metrics will be more efficient and accurate to express the connection situation among a group of users.

From the QoS point of view, the multiparty communication services not only require the absolute QoS support but also the relative QoS support. The relative QoS means the difference between absolute QoS of all users. Directly using the end-toend metric cannot present the relative QoS situation. If we use the variations of all users' end-to-end parameters, we can have new metrics to measure the difference of the absolute QoS and hence provide the threshold value of relative QoS that a multiparty service might demand. A very good example of the high relative QoS requirement is the online gaming. A very light worse delay will result in failure in the game. We have to use the new metrics to define exactly how small the relative delay the online gaming requires. There are many other services, e.g. online biding, online stock market, etc., need a rule to judge the relative QoS requirement. Therefore, we can see the importance of new metrics to feed this need. Two groups of parameter are proposed in this stage.

To conveniently define new metrics, we call all of the users in the same multiparty communication a user group. This user group should not be mixed with the multicast user group. Group members could use either pure unicast or multicast to communicate or mixed, i.e. some of the users in the group could use unicast while others use multicast.

When we talking about a new metrics we always have an observe point that is one of the users in the group. We classified the new metrics into two groups based on the fact that one user could be either a source or a receiver. Therefore, one group metrics will describe the QoS of the traffic coming out from the group to one particular user and another group describes the QoS going into the group. We name them as one-to-group parameters and group-to-one parameters.

These new proposed parameters are established on the base of the one-way metrics defined in the corresponding RFCs in the IPPM working group. And no modification should be added to those one-way metrics in any aspects.

\section{A. One-to-group Parameters and Metrics}

One-to-group parameters are defined to measure the QoS in the view of a group user. Two subset parameters are introduced:

1. One-to-group (algorithm) mean

a) One-to-group mean delay

b) One-to-group mean jitter

c) One-to-group mean packet lost rate

2. One-to-group variation

a) One-to-group delay variation

b) One-to-group jitter variation

c) One-to-group packet loss rate variation

The one-to-group parameters are measured based on only one source in a multiparty communication group. Whenever we say one-to-group parameter, we should associate it with a source. The Figure 1 shows this concept.

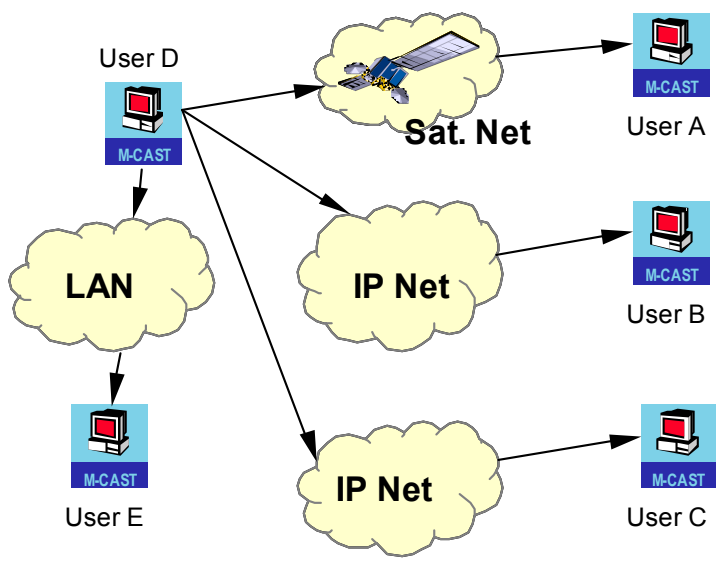

Figure 1: One-to-group measurement scenario example

In Figure 1 user A, B, C, D and E belong to the same multicast group. User D is the only active source in the multicast group when measuring the one-to-group parameters. User B and C are connected with user D through terrestrial IP network, user $\mathrm{E}$ are in the same LAN with user $\mathrm{D}$, and user $\mathrm{A}$ are connected with user D using a satellite network. The oneto-group parameters measured in this scenario should be associated with user D.

1) One-to-group (Arithmetic) Mean

One-to-group mean parameters are trying to measure the overall QoS for a multiparty communication group. The 
definition of the One-to-group mean is the mean of a one-way parameter, such as one-way delay, one-way jitter and packet loss rate, measured simultaneously on all of the group members except of the active source. The word "simultaneously" implies the one-way parameter should be measured based on the same sample interval at each user.

The One-to-group mean parameters can be calculated as:

$$
P_{\text {OGM_para }}=\frac{\sum_{i=1}^{N} P_{i}}{N}
$$

where $P_{O G M \text { para }}$ is the One-to-group mean parameter, $P_{i}$ is the corresponding one-way parameter. $N$ is the number of the users except the active user in the group during the sampling interval. "para" means the one-way parameter's name such as delay, jitter and packet loss rate.

\section{Metric Name:}

Type-P-One-to-group-Mean-Parameter

The "Parameter" could be any one of the one-way parameter defined in IPPM including delay, jitter and packet loss rate.

\section{Metric Parameters:}

- Src, the IP address of a source

- Grp, the multicast group address is for multicast or empty for non-multicast

- $\quad \mathrm{M}$, a derived value corresponding to one-way parameter

\section{Metric Units:}

The value of a Type-P-One-to-group-Mean-Parameter is depends on what one-way parameter is used. It should be the same corresponding to the one-way metrics defined in IPPM.

\section{Methodologies:}

As the metric is derived from the corresponding one-way metric, the methodology to obtain those one-way parameters can be referred to the corresponding RFCs. We only discuss the methodology to derive One-to-group mean metric from one-way parameter without consideration of details on synchronization, test packetizing, time and etc.

1. Simultaneously measure the interested one-way parameters, one-way delay, one-way jitter or packet loss, on all of the receivers in a multiparty communication group when there is only one source active.

2. Calculate the mean of one-way metric value using equation (1) to obtain the One-to-group mean metric for this source. The question of when to calculate the One-to-group mean metric will be discussed later.

3. Change the active source and repeat the step 1 and 2 until all of the group members have been active as sources.

2) One-to-group Variation

One-to-group variation metrics are trying to measure how the QoS varies among all of the users in a multiparty communication group relative to one source. The word "variation" in this document is the population standard deviation. The definition of the One-to-group variation is the population standard deviation of a one-way parameter, such as one-way delay, one-way jitter and packet loss rate, measured simultaneously at all of the group members except of the active source. Therefore, we can have One-to-group delay variation, One-to-group jitter variation and One-to-group packet loss rate variation. The word "simultaneously" implies the one-way parameter should be measured based on the same sampling interval at each user. Considering the case shown in Figure 1 as an example, when $\mathrm{D}$ is active, we simultaneously monitor a set of packets from $P_{1}$ to $P_{n}$ on all of the rest 4 users respectively. Then, the interested one-way parameter of these packets is calculated for each of user. The corresponding One-to-group mean metric could be calculated based on the one-way parameter. Finally, we calculate the variation of these 4 values of the one-way parameter measured on 4 receivers as the One-to-group variation parameter for this scenario. The One-to-group variation parameter can be denoted by $P_{O G V \text {-para }}$, where the symbol "para" means the oneway parameter's name such as delay, jitter and packet loss rate. And the calculation should be:

$$
P_{O G V \text { - para }}=\sqrt{\frac{\sum_{1}^{N}\left(P_{i}-P_{O G M-\text { para }}\right)^{2}}{N}}
$$

where $P_{i}$ is the one-way parameter value (delay, jitter and packet loss rate) and $P_{O G M \text { para }}$ is the corresponding One-togroup mean parameter value. $N$ is the number of the receivers.

\section{Metric Name:}

Type-P-One-to-group-Variation-Parameter The "Parameter" could be any one of the one-way parameter defined in IPPM including delay, jitter and packet loss rate.

\section{Metric Parameters:}

- $\quad$ Src, the IP address of a source

- Grp, the multicast group address is for multicast or empty for non-multicast

- $\quad \mathrm{V}$, a derived value corresponding to one-way parameter

\section{Metric Units:}

The value of a Type-P-One-to-group-Variation-Parameter is depends on what one-way parameter is used. It should be the same corresponding to the one-way metrics defined in IPPM.

\section{Methodologies:}

As the One-to-group variation parameter metric has to be derived on the base of the group mean metric, we have to calculate the One-to-group mean metric first. So the methodology become simple inheriting from the one defined for the One-to-group mean metric.

1. Find out the One-to-group mean parameters

2. Calculate the One-to-group variation parameters using equation (2). 
3. Repeat the step 1 and 2 for all users in the same multiparty communication group.

\section{B. Group-to-one Parameter and Metrics}

Group-to-one parameters are defined to measure the QoS in the view of one multiparty communication user with respect to the fact that this user is receiving from more than one source in the group. Similar to the one-to-group parameters, two subset parameters are proposed:

1. Group-to-one member (arithmetic) mean
a) Group-to-one mean delay
b) Group-to-one mean jitter
c) Group-to-one mean packet loss rate

2. Group-to-one variation
a) Group-to-one delay variation
b) Group-to-one jitter variation
c) Group-to-one packet loss rate variation

The group-to-one parameters are measured based on only one receiver in a multiparty communication group. Whenever we say group-to-one parameter, we should associate it with the receiver. The Figure 2 shows this concept.

Figure 2 shows almost the same information as Figure 1. The difference is in Figure 1, user D is the receiver who received data from all of the rest group members simultaneously or consequently. The group-to-one parameters measured in this scenario should be measured and associated with user D.

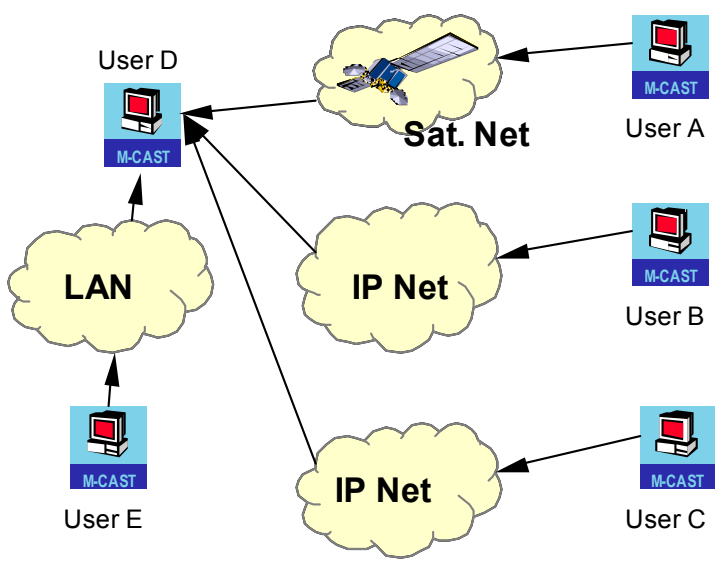

Figure 2: Group-to-one measurement scenario example

In the following sections, we will define these parameters and their metrics. You will find the definitions are very similar to one-to-group parameters. One might question on if we need to have separately definitions of one-to-group and group-toone parameters. The answer is positive and we will discuss it after the definition.

\section{1) Group-to-one (arithmetic) Mean}

Group-to-one mean parameters are trying to measure the QoS of a multiparty communication group received by one user.
The definition of the Group-to-one mean parameter of a user is the mean of a one-way parameter, such as one-way delay, one-way jitter and packet loss rate, measured on that user when it simultaneously receiving data from all the rest users in the group. The word "simultaneously" implies the one-way parameter should be measured based on the same sampling interval on the measured user. The Group-to-one mean parameters can be calculated as:

$$
P_{\text {GOM_para }}=\frac{\sum_{i=1}^{N} P_{i}}{N}
$$

where $P_{G O M_{-} \text {para }}$ is the Group-to-one mean parameter, $P_{i}$ is the corresponding one-way parameter from each of the source to the measured user. $N$ is the number of the users except the measured user in the group during the sampling interval. "para" means the one-way parameter's name such as delay, jitter and packet loss rate.

\section{Metric Name:}

Type-P-Group-to-one-Mean-Parameter

The "Parameter" could be any one of the one-way parameter defined in IPPM including delay, jitter and packet loss rate.

\section{Metric Parameters:}

- Dst, the IP address of a receiver

- Grp, the multicast group address is for multicast or empty for non-multicast

- $\mathrm{M}$, a derived value corresponding to one-way parameter

\section{Metric Units:}

The value of a Type-P-Group-to-one-Variation-Parameter is depends on what one-way parameter is used. It should be the same corresponding to the one-way metrics defined in IPPM.

\section{Methodologies:}

As the group-to-one mean parameter metric also derived on the base of the corresponding one-way parameter metric, we still only discuss the methodology to derive Group-to-one mean metric from one-way metric without consideration of details on synchronization, test packetizing, time and etc..

1. Simultaneously measure the interested one-way parameters, one-way delay, one-way jitter or packet loss, on the measured user while all of the rest of users in the multiparty communication group sending data to it. All the one-way parameter should be measured based on the source and destination pair.

2. Calculate the mean of one-way metric value using equation 3 to obtain the Group-to-one mean metric for the measured user. The question of when to calculate the group-to-one mean metric will be discussed later.

3. Change the active source and repeat the step 1 and 2 until all of the group members have been measured. 


\section{2) Group-to-one Variation}

Group-to-one variation metrics are trying to measure how the QoS varies at one user in the multiparty communication group when the rest of users sending data to it. The definition of the Group-to-one variation is the population standard deviation of a one-way parameter, such as one-way delay, one-way jitter and packet loss rate, measured at one user in a multiparty communication group while all of the rest group members sending data simultaneously to it. Therefore, we can have Group-to-one delay variation, Group-to-one jitter variation and Group-to-one packet loss rate variation. The word "simultaneously" implies the one-way parameter should be measured based on the same sample interval at the measured user. Considering the case shown in Figure 2 as an example, when $\mathrm{D}$ is chose as the measured user, we simultaneously monitor a set of packets from $P_{1}$ to $P_{n}$ sent by each of the rest 4 users respectively. Then, the interested one-way parameter of these packets is calculated for each pair of users, i.e., $\mathrm{D}$ and $\mathrm{A}, \mathrm{D}$ and $\mathrm{B}, \mathrm{D}$ and $\mathrm{C}$ and $\mathrm{D}$ and $\mathrm{E}$. The corresponding Groupto-one mean metric could be calculated based on the one-way parameter. Finally, we calculate the variation of these 4 values as the Group-to-one variation parameter for this scenario. The One-to-group variation parameter can be denoted by $P_{G O V-p a r a}$, where the symbol "para" means the one-way parameter's name such as delay, jitter and packet loss rate, and calculation should be:

$$
P_{\text {GOV-para }}=\sqrt{\frac{\sum_{1}^{N}\left(P_{i}-P_{\text {GOM-para }}\right)^{2}}{N}}
$$

where $N$ is the total user number in the multiparty communication except the measured one and $P_{i}$ is the oneway parameter for each of the $N$ users.

\section{Metric Name:}

Type-P-Group-to-one-Variation-Parameter

The "Parameter" could be any one of the one-way parameter defined in IPPM including delay, jitter and packet loss rate.

\section{Metric Parameters:}

- Dst, the IP address of a receiver

- Grp, the multicast group address is for multicast or empty for non-multicast

- $\mathrm{V}$, a derived value corresponding to one-way parameter

\section{Metric Units:}

The value of a Type-P-Group-to-one-Variation-Parameter is depends on what one-way parameter is used. It should be the same corresponding to the one-way metrics defined in IPPM.

\section{Methodologies:}

The methodology can be simply inherited from the one defined for the Group-to-one mean metric as:

1. Find out the Group-to-one mean parameters
2. Calculate the Group-to-one variation parameters using the equation 4

3. Repeat the step 1 and 2 for all users in the same multiparty communication group

\section{REASONS FOR TWO GROUPS OF SIMILAR PARAMETERS}

As we mentioned in the beginning of section III, the definitions of One-to-group parameters and Group-to-one parameters are very similar. There are reasons we should separately define them. Firstly, it is because of the metric parameter definition. The One-to-group metrics have a common parameter, $S r c$, the IP address of the active source during the measurement interval. It must be changed to Dst parameter for the Group-to-one metrics to present the measured user. It's not like the case for the one-way parameter measurement where the destination and the source are single host in the same level. They can be exchanged in the measurement without any difficulty. Therefore one metric is enough for measurement between one pair of hosts. In the multiparty communication, the source and the destination cannot be exchanged because one of them presents more than one user. We have to define two metrics for the measurement for two directions. For instance, if user $\mathrm{A}$ and user $\mathrm{B}$ communicates with each other, the one-way delay metric can be used for both direction traffics by exchanging the $S r c$ and Dst parameter [4]. However, if user C joins their communication, we have to user the proposed new metrics to measure the QoS for the multiparty communication. The One-to-group mean delay metric and the One-to-group delay variation can show clearly the QoS received by user A and user B in the group relative to user C. We cannot use the same metrics to measure the QoS received by $\mathrm{C}$ relative to both user A and user B by simply exchanging the Src and Grp parameter in the metric because of the methodology described for One-to-group parameter.

Secondly, we should define Group-to-one and One-to-group separately because of the transporting technologies used for multiparty communications. There might be the coexistence of both unicast and multicast. One host in a multiparty communication group might use unicast to receive data from other hosts and user multicast to send data to the others. The delay of each direction would be different due to the difference of the transport technologies. If we can say that for one-to-one communications, delays for both directions can be approximately the same, we might not have the same conclusion for the multiparty communications. Therefore, we need two groups of metric to describe the network situation regarding the traffic direction.

\section{PARAmeter CAlculation}

With the definition of the parameters, we have to decide when to do the calculation. Here we take the One-to-group mean 
metric calculation as an example. Basically, there are three ways to do so. The first way is to do the calculation based on each packet arrival. The active source sends packet one by one with sequence number in the packet headers so that all receivers could identify each packet. The One-to-group mean calculation is executed for each packet received by all receivers. The resulted metric is similar to the singleton metrics defined for one-way parameters corresponding to every packet received by all users. It will provide the most accurate record of the group mean during a sampling interval with the heaviest calculation overhead.

The second way to calculate the One-to-group mean is to use the mean of one-way parameter rather than the parameter itself. The calculation could be scheduled to be executed periodically. For instance, it can be triggered for every $\mathrm{T}$ seconds. During the $\mathrm{T}$ seconds, all one-way parameters measured have to be recorded at each receiver. At each $\mathrm{T}$ second, the mean of the recorded parameter will be calculated first at each receiver and used as $P_{i}$ in equation (1) to calculate the One-to-group mean metric value. This way can reduce the heavy calculation overhead required by the first one. However, it would provide less detailed information and need more storage space to record one-way parameters for more than one packet.

The third way to calculate the One-to-group mean metric is to mix the previous two ways together. We periodically calculate the One-to-group mean parameter using directly the corresponding one-way parameter metric value rather than using its mean. For instance, the calculation can be prearranged to be triggered for every $\mathrm{T}$ seconds. The receivers don't need to record the one-way metric value for all of the packets received during each $\mathrm{T}$ seconds. We would calculate the One-to-group mean metric value at each $T$ second using the corresponding one-way parameter of the latest received packet. Therefore, the One-to-group mean metrics of all receivers calculated at the same time would not be for the same packet. However, that would not affect engineers to use these metrics because they can still present the network situation at each $\mathrm{T}$ second without regarding to packets. Hence, the sequence number seems not necessary for One-to-group mean delay and jitter metrics. However, it still has to be added to the test packets to notify the packet loss. By calculating the One-to-group mean metrics in this way, we can overcome the requirement of big storage space on each receiver and the calculation overhead. One point has to be mentioned here is the calculation of the One-to-group mean packet loss rate. Because the packet loss rate itself is a statistic parameter for a certain measurement interval, we have to use the second way to calculate the One-to-group mean packet loss rate.

Clearly, the One-to-group mean calculation period $\mathrm{T}$ is a very important factor in the implementation of the measurement. If it is too small, we will not save any calculation overhead. If it is too big, we might loss most of the network situation information. And it might be different for various applications as well. How to find a proper $\mathrm{T}$ might be vary for different applications. It is out of the scope of this paper.

\section{ANALYsis OF THE Possible MEASUREMENT ERRORS}

We are not going to discuss errors caused by the measurement of the one-way parameters in this document because they can be found in the corresponding RFCs. We have to discuss the errors introduced by the proposed metrics in this document. The reason of these errors is the packet loss in the network. If a packet is lost, its delay might be hidden from the result.

When we discussed about when to calculate the proposed metrics, we gave three ways. The first way provides one-way parameter metrics corresponding to packets. That means for each packet we can find one metric value for it. Then error caused by packet loss can then be easily sorted out before we calculate the Group-to-one and One-to-group parameters.

However, for the other two ways, we either use a mean to present the interested one-way parameter or the last packet received by the measurement point during a period of time. If there are any packets lost in the period of time, they will be ignored by the calculation of the multiparty communication parameters. For instance, we do the calculation of the multiparty communication parameters for every $\mathrm{T}$ seconds. Then for the second way, the mean of the one-way delay in a $\mathrm{T}$ second could be infinity if any packets lost during that $\mathrm{T}$ seconds and infinity is a valid metric value for the one-way delay metric. Our Group-to-one and One-to-group mean parameters and variation parameters could be infinity after calculation. This infinity doesn't mean anything in terms of relative delay for multiparty communication. We should not have the conclusion that during that $\mathrm{T}$ seconds, users in the group suffered significantly different delay.

For the third way where we only use the latest packet received during a $\mathrm{T}$ seconds, if all of the packets were lost during the $\mathrm{T}$ seconds, which is quite possible since $T$ could be a very short time, the one-way metric value we use to calculate the multiparty communication parameters will be the one for the last packet receiver in the last $\mathrm{T}$ seconds. Clearly, the result will not reflect any information of the network situation during this $\mathrm{T}$ seconds and therefore, it becomes an error.

The calibration can be done by using more sophisticated way to calculate the multiparty communication parameters. For instance, we can ignore all the one-way metrics with infinity value when we calculate the multiparty communication parameters for the second calculation way. We can find out which $\mathrm{T}$ seconds suffers from the packet lost and do not calculate the multiparty communication parameter for it in the third way. There might be other methods to calibrate the errors, which we did not discuss here. As long as they can avoid leading us to the wrong analysis, they can be implemented in the application. 


\section{CONCLUSIONS}

This paper had a brief introduction on the traffic measurement and one-to-one QoS parameters recommended by IPPM working group. A set of new parameters and their metrics derived from those one-to-one parameters were proposed to describe network performance for multiparty communications. These parameters are also necessary to be used to measure and judge the relative QoS requirements for multiparty communications.

Three possible means to calculate these proposed parameters were also discussed in terms of their advantages and disadvantages. Some possible measurement errors that might be met in the calculation were also presented with possible solutions.

These parameters are derived from the IPPM parameters and, hence, no modification should be made to those based RFCs in any aspect. The corresponding metrics are presented in terms of metric name, metric parameters, metric unit and measurement methodologies based on the criteria recommended by the IPPM working group and a similar internet draft has been submitted to the IETF for comments.

\section{REFERENCE:}

[1] Kenneth C. Glossbrenner, "Internet Protocol Data Communication Service - IP Packet Transfer and Availability Performance Parameters," Approved Text for New Recommendation I.380, Specification and Allocation of Internet Service Performance (T1A1-14), ITU-T Study Group 13, May 3, 1999.

[2] V. Paxson, G. Almes, J. Mahdavi, M. Mathis, "Framework for IP Performance Metrics," IETF RFC 2330, May 1998.

[3] J. Mahdavi, V. Paxson, "IPPM Metrics for Measuring Connectivity," IETF RFC 2678, September 1999.

[4] G. Almes, S. Kalidindi, M. Zekauskas, "A One-way Delay Metric for IPPM,” IETF RFC 2679, September 1999.

[5] G. Almes, S. Kalidindi, M. Zekauskas, “A One-way Packet Loss Metric for IPPM,” IETF RFC 2680, September 1999.

[6] G. Almes, S. Kalidindi, M. Zekauskas, "A Round-trip Delay Metric for IPPM,” IETF RFC 2681, September 1999.

[7] R. Koodli, R. Ravikanth, Axiowave, "One-way Loss Pattern Sample Metrics," IETF RFC 3357, August 2002.

[8] C. Demichelis, P. Chimento, "IP Packet Delay Variation Metric for IP Performance Metrics," IETF RFC 3393, November 2002.

[9] S. Blake, D. Black, M. Carlson, E. Davies, Z. Wang, and W. Weiss, "An Architecture for Differentiated Services," IETF RFC 2475, December 1998. 